\title{
Coherence and connections in teachers' mathematical discourses in instruction
}

\begin{abstract}
Authors:
Hamsa Venkat ${ }^{1}$

Jill Adler ${ }^{1}$
\end{abstract}

Affiliations:

${ }^{1}$ School of Education,

University of the

Witwatersrand, South Africa

Correspondence to:

Hamsa Venkat

Email:

hamsa.venkatakrishnan@

wits.ac.za

Postal address:

Room 2, WMC Corridor,

Wits School of Education,

St Andrews Road, Parktown

2050, Johannesburg,

South Africa

Dates:

Received: 13 Aug. 2012

Accepted: 21 Oct. 2012

Published: 11 Dec. 2012

Re-published: 21 Dec. 2012

Note:

This article was re-published with a correction made on the last equation on this page.

How to cite this article: Venkat, H., \& Adler, J. (2012) Coherence and connections in teachers' mathematical discourses in instruction. Pythagoras, 33(3), Art. \#188, 8 pages. http://dx.doi. org/10.4102/pythagoras. v33i3.188
In this article, we share our combination of analytical concepts drawn from the literature with a set of grounded framing questions for thinking about differences in the nature of coherence and connections in teachers' mathematical discourses in instruction (MDI). The literaturebased concepts that we use are drawn from writing focused on transformation activity as a fundamental feature of mathematical activity. Within this writing, the need for connections between stated problems and the representations introduced and subsequently produced through transformation steps are highlighted. Drawing from four empirical episodes located across primary and secondary mathematics teaching, we outline a set of framing questions that explore coherence and connections between these concepts, and the ways in which accompanying explanations work to establish these connections. This combination allows us to describe differences between the episodes in terms of the nature and degree of coherence and connection.

\section{Introduction}

Teachers' mathematical discourses in instruction (MDIs), essentially the mathematical aspects of what teachers say, do and write as they interact with learners in mathematics classrooms, are a key feature of classroom practice. Typically, these MDIs include a problem, a selected representation that is subsequently transformed, and explanations and justifications for the representations selected and transformations performed. Our interest in this article is in developing a language that can be used to describe a range of MDIs. This interest is driven by our need to understand MDIs that seem to us to disrupt coherence and connection in mathematical text in a range of ways, and thus impact on what mathematics is made available to learn.

Transformation of representations, through manipulations within and across different representation forms, is a central feature of mathematical activity (Duval, 2006) and, therefore, of MDIs. Solving a problem in school mathematics often involves a set of steps through which one representation is transformed into another. For example, completing the square is comprised of a series of transformation steps that can act upon a quadratic function as input representation, if the stated problem is to find the turning point of the function. Consider the problem:

Find the turning point of $f(x)=x^{2}-8 x+9$

The first step to solving this stated problem could be to recognise that rewriting a quadratic expression as a perfect square, plus or minus some constant, allows us to 'see' vertical and horizontal shifts with respect to the parent function, and so the turning point, more easily. We would thus rewrite the function in the form $f(x)=a(x-p)^{2}+q$ by completing the square:

$$
\begin{aligned}
f(x) & =x^{2}-8 x+9 \\
& =x^{2}-8 x+16-16+9 \\
& =(x-4)^{2}-7
\end{aligned}
$$

What is important for this transformation activity ${ }^{1}$ is that within the MDI, the input representation introduced, the representations produced through transformation activity, and the accompanying explanations connect with each other and cohere with the stated problem. Our observations, across primary and secondary classrooms within our respective projects, suggest that such coherence or connection is frequently, but varyingly, disrupted within MDIs.

In this article, we share our development of an empirically derived analytical language (elements of which are italicised above) that allows us to make visible a range of disruptions to connection 1.Our use of the term 'transformation activity' coheres with Duval's (2006) use of the term, which refers to transformations within and
across registers. It is more inclusive than Kieran's (2004) notion of transformation, which is restricted to transformation of algebraic expressions whilst maintaining equivalence across these manipulations. 
and coherence that come into play across four contrasting teaching episodes. We focus here on input objects, transformational activity and accompanying explanations in order to 'see into' the micro-level production of mathematics in classrooms through describing differences in the nature and degree of coherence and connection and to consider the consequences for what is made available to learn. This micro-level focus on specific episodes within lessons follows our observation of the occurrence of disruptions at this level, rather than at the broader level of lessons or lesson sequences that have been taken up in prior research (e.g. Sekiguchi, 2006).

In order to present our thinking on making aspects of connection and coherence visible within transformation activity, we begin with a brief overview of the literature. We draw on writing focused on transformation activity and representations as these actions and objects are at the centre of all our episodes and, as noted already, at the heart of mathematical activity more generally. We also summarise evidence that points to the shortcomings that characterise practices in which transformation steps are emphasised at the expense of gaining understanding of the representations they act upon. From this review, we outline the key concepts that we found helpful in beginning to pull apart some of the range of procedural practices that we were working with. Centrally, we home in on the stated problem, the selected input representation, subsequent sequences of transformation steps, and the interim and final representations produced in this sequence. These concepts are all covered in the literature we review.

Somewhat absent in this literature is a focus on the MDIs that accompany transformation activity. Teaching involves the giving of accompanying explanations alongside transformation and so, unlike the (often predictably) piecemeal learner discourses that are in focus in much of the literature on transformation-oriented activity, one expects MDIs to be both coherent and connected, and to provide some of the rationales for the representations selected and transformation activity that is enacted. As noted above however, we see this expectation disrupted relatively frequently, and in a range of different ways. In order to consider the nature of these disruptions to coherence, we use a tentative set of framing questions, drawn from our grounded analysis of the episodes presented in this article, to analyse and differentiate the transformation activity in four selected teaching episodes. This could be criticised as somewhat circular: developing grounded framing questions from a dataset, and then using them to analyse the same dataset. Our aim in doing this is to share this set of literature-drawn concepts and grounded framing questions in order to start conversations across the mathematics teaching and teacher education communities that can help to build a more robust language for thinking about what constitutes coherence and connection within mathematics teaching. We have already been through several iterations of concepts and framing questions, and have seen that our current formulation can be applied to a significantly broader group of episodes that we have encountered.

\section{Describing transformation activity - a literature review}

Duval (2006), from a semiotic perspective, describes mathematical activity as comprised by the transformation of one semiotic representation into another. For Duval, mathematical transformations can happen within, or across, registers: encompassing natural language, numeric, symbolic and algebraic notations, graphical representations, geometric figures and tabular presentations. For our purposes, the focus is on the representations and transformations selected and produced within transformation activity, the turning of one representation into another, either within or across registers.

Paying attention to the representations selected and produced through transformation activity is described by Haapasalo and Kadijevich (2000), cited in Haapasalo (2003), as important within transformation activity underlain by strong procedural knowledge. Strong procedural knowledge, for them, involves:

dynamic and successful utilization of particular rules, algorithms or procedures within relevant representation forms. This usually requires not only the knowledge of the object being utilized, but also the knowledge of format and syntax for the representational system(s) expressing them. (p. 98)

This synchronous attention to both representational objects and transformation techniques is often described as lacking in school mathematics.

An important second thread in the mathematics education literature is highly critical of the ways in which transformation activity has come to be configured within classrooms. Artigue (2011), in her article for UNESCO on the challenges of extending basic mathematics and science education for all students, refers to international surveys to describe how schooling is very often unstimulating because the teaching of mathematics is framed by:

- formal teaching, centred on learning techniques and memorizing rules whose rationale is not evident to the pupils

- pupils [who] do not know which needs are met by the mathematics topics introduced or how they are linked to known concepts. (p. 21)

Implied within Artigue's formulation is a situation within which representations tend to be backgrounded, whilst transformation techniques are foregrounded. The need for a sense of the 'problem' that drives the selection of representations and the transformations enacted on them (the 'raison d'etre') in coherent ways is highlighted. Drawing from the analytical work of Duval, and the critiques presented here, we see that the concepts of stated problem, input and subsequent representations produced through transformation activity are highlighted as fundamental to mathematical activity.

The international literature base is replete with evidence of the consequences of pedagogies based on these kinds of practices for mathematical learning (Thompson, Philipp, 
Thompson \& Boyd, 1994). De Lima and Tall (2008) provide evidence of learners enacting transformations of algebraic representations based on the 'embodied actions they perform on the symbols, mentally picking them up and moving them around, with the added "magic" of rules' (p. 3). Such actions indicate inattention to the representations being operated on and the syntax of the registers these representations are located in; they thus frequently produce incorrect answers. Learner performance in South Africa across all phases attests to similar problems (Department of Basic Education, 2011a, 2011b). More problematically, there is evidence in South Africa of some of these actions occurring in the context of teaching rather than at the level of learner working (Mhlolo, Venkat \& Schafer, 2012).

This leads to the need to focus on MDIs. Instructional explanation has been described as a 'commonplace' of mathematics teaching (Leinhardt, 2001), and described in terms of the 'orchestrations of demonstrations, analogical representations and examples' (Leinhardt, Zaslavsky \& Stein, 1990). The word 'orchestration' points to the coherence and connection between problems and representations that we are focusing on, but does not, in itself, provide descriptors of what might constitute 'good' orchestration. Similarly, whilst coherence was identified as a characteristic seen more frequently in some Asian countries within the TIMMS-video data (Hiebert et al., 2003), what constitutes this coherence within teaching is not detailed. Rowland (2012), in using the distinction made in Leinhardt's (2001) work between disciplinary and instructional explanations, notes that deductive reasoning characterises the former, whilst the need to 'help students learn, understand and use knowledge' through the use of 'carefully devised analogy' that render explanations 'more accessible and more palatable' (p. 59) is key to instructional explanations. In this more learningfocused category, there is a need to 'establish' rather than 'state' deductive connections, in order to support learner understanding of critical links. Thus, we looked at the nature of both disciplinary and instructional explanations in MDIs through framing questions that would allow us to analyse differences in connection and coherence between our episodes.

In the teaching episodes we present in this article, all drawn from previously published work in the South African landscape, practices are exemplified at a range of problematic levels, beginning in Artigue's terrain (backgrounding of problems and representations and foregrounding of transformations), and moving to significantly deeper problems in relation to coherence and connection. Our framing questions allowed us to disaggregate episodes in ways that provided some windows into understanding what constitutes coherence and connection.

\section{Concepts and framing questions}

Mathematical processing begins with a stated problem (SP). Stated problems have to be solved through the introduction of an initial input representation, which could be a symbolic statement, visual representation, et cetera. In each of our episodes, we refer to this introductory offering to be operated upon as the input representation (IR). Transformation activity (TA), constituted by the transformation steps enacted on this input representation, and the interim representations produced, then ensues. Given our focus on teaching, these are associated with accompanying explanations from the teacher.

We focus on the detail of transformation steps enacted and the interim representations produced through these transformations, noting, in the first instance, whether these representations retain connection to the input representation. It is worth noting here that algorithms in mathematics do sometimes break this connection at interim stages, and reinstate it at the final stage; the long division algorithm provides a well known example of this (see Long [2005], for an elaboration on the differences between procedures and algorithms, and the deep mathematical structures underlying algorithms). We note this point to emphasise that these breaks in connection need not be innately problematic if the scope of application to representations and the mathematics underlying the transformation sequence are considered within the accompanying explanation. We therefore look at teacher explanations for whether or how they establish connections between representations and transformations, coherence with the stated problem, and reference to the scope of application and mathematical structure of the transformation being dealt with.

The framing questions are presented below:

- Does the input representation cohere with the stated problem by providing an appropriate representation to transform?

- Does transformation activity produce representations that connect in mathematically defensible ways with the input representation? Does this happen (1) across all interim representations or (2) at final representation?

- Does transformation activity, linked with the teacher's accompanying explanation, serve to establish connections between its steps at each stage and the input representation?

Our questions reflect more basic notions about transformation activity than have been dealt with in the existing literature that deals with both cognition and semiotics (Duval, 2008). In the literature we have summarised, the notion of coherence and connection in teachers' selections and transformations of representations is largely assumed, and critique focuses on the absence of rationales for the transformations selected. Our episodes suggest the need to suspend this assumption and focus on the detail of problem-representation-transformation connections as they play out in teaching. Firstly, we look for whether the input representation presented to transform coheres with the stated problem. We then ask questions about how interim and final representations produced within transformation activity connect to the input representation. This often involves transformation activity that produces representations that maintain equivalence between the representation acted on 
and the representation produced. At a deeper level though, we can also ask whether this transformation activity, linked with the accompanying explanation, establishes connections with the input representation rather than simply assuming, or stating, the connection.

In the next section, we present episodes drawn from previously published work.

\section{Episodes \\ Episode 1}

This episode was reported in Adler and Pillay (2007) and Adler (2012) and is drawn from a study by Pillay (2006). Nash (pseudonym), a secondary school teacher, is described as well respected in his school, at which student performance in Grade 12 national mathematics examinations was considered successful. The episode below is taken from the third in a unit of eight consecutive lessons on linear functions in a Grade 10 class. In Lesson 1 and Lesson 2, Nash had dealt with drawing the graph of a linear function first from a table of values, and then using the gradient and $y$-intercept method. All the linear function examples that were worked through in the first two lessons were in the standard form $y=m x+c$.

In Lesson 3, he moves on to demonstrate how to draw the graphs of functions that are not expressed in standard form. He begins with a few examples (e.g. $2 y-4 x=2$ ), using the gradient-intercept method, and the manipulative work needed to get these into the standard form $y=m x+c$. This serves as motivation for the greater simplicity of the dual intercept method for drawing straight-line graphs. $\mathrm{He}$ returns to a function they had worked on, $3 x-2 y=6$, and begins a discussion of 'dual' meaning 'two', eliciting from learners that the two intercepts are where the graph cuts the $x$ and $y$ axes. He demonstrates how to find the coordinates of the $y$-intercept by calculating the value of $y$ when $x=0$ and, similarly, the coordinates of the $x$-intercept. He writes $(0 ;-3)$ and $(2 ; 0)$ on the chalkboard and proceeds to sketch the axes, explaining how you can 'estimate' where the points are on each of the axes. He plots the two points and continues:

Nash:

... all I have to do join these important points and I got [states and labels the line] $3 x-2 y=6-$ wasn't that much easier? - there's less mathematics to do [points to the calculations from the previous question] [than] when you come to write it in $y$ form. Simple, first make your $x$ equal to zero that gives me my $y$-intercept. Then the $y$ equal to zero gives me my $x$-intercept. Put down the two points - we only need two points to draw the graph.

Then a learner asks a question:

Learner 1: You don't need all the other parts?

Nash: $\quad$... What's important features of this graph? ... we can work out ... from here [points to the graph drawn] we can see what the gradient is ... is this graph a positive or a negative?
Learners: [Chorus] Positive.

Nash: It's a positive gradient ... we can see there's our $y$-intercept, there's our $x$-intercept [points to the points $(0 ;-3)$ and $(2 ; 0)$ respectively].

After a brief discussion on the labelling of points on a graph, Learner 2 and Learner 3 ask Nash:

Learner 2: Sir, is this the simplest method, sir?

Learner 3: How do you identify which side must it go, whether it's the right-hand side. [Nash interrupts]

Nash: [Response to Learner 2] You just join the two dots.

Learner 2: That's it?

Nash: $\quad$ Yeah ... the dots will automatically ... if it was a positive gradient it will automatically ... if this was [refers to the line just drawn] negative ... that means this dot [points the $x$-intercept] will be on that side [points to the negative $x$ axis] ... because if the gradient was negative, how could it cut on that side? [Points to the positive $x$ axis]

Learner 2: $\quad$ Is this the simplest method sir?

Nash: The simplest method and the most accurate

Learner 4: Compared to which one?

Nash: $\quad$ Compared to that one [points to the calculation of the previous question where the gradient and $y$-intercept method was used] because here if you make an error trying to write it in $y$ form ... that means it now affects your graph ... Whereas here [points to the calculations he has just done on the dual intercept method] you can go and check again ... you can substitute ... if I substitute for 2 in there [points to the $x$ in $3 x-2 y=6$ ] I should end up with 0 .

Our interest here is in how the teaching of the dual intercept procedure unfolds through transformation activity and its accompanying explanation. Nash presents a function $3 x-2 y=6$ (an input representation) where the stated problem is to draw the graph of this line. This representation does cohere with the stated problem, and the preceding activity also shows that producing a line using the transformation sequences that have already been taught can be done, but is long-winded, making an alternative method useful. Thus the need for an alternative transformation sequence is motivated in Nash's accompanying explanation. He then presents the steps to follow to obtain the coordinates of each of the intercepts. All the interim representations and the final representation do connect appropriately and cohere with the stated problem.

Whilst Nash's advocacy of the dual intercept method in all instances blocks some of the flexibility that a more selective match between stated problem and input representation would allow, and also does not provide ways of dealing with special cases (horizontal, vertical lines and lines through the origin), important features of the input and subsequent representations and their connections do remain in focus across Nash's lesson sequence. For example, the fact that the gradient can be derived from the application of the dual intercept transformation steps to a linear equation to produce the associated graph is explained in instructional terms, using the move between algebraic and graphical representations to emphasise how given information can be used to derive missing information. 


\section{Episode 2}

In this episode, drawn from Askew, Venkat and Mathews (2012), a Grade 2 teacher is working on missing addend problems using a wheel representation with three concentric circles: 7 written on the inner circle, and the numbers $0-7$ placed in random order around the outermost circle in separate sectors. Askew et al. state:

The task explained by the teacher was to fill in the intermediate circle with the numbers that needed to be added to the outer rim numbers of the wheel to make the number 7. (p. 29)

The stated problem of the lesson, indexed by the title on the board Hlanganisa ('Addition' in Zulu), is for the class to fill in the numbers that need to be added to the numbers on the edge to make 7. Initial answers from some of the children indicate that they are interpreting the task in terms of addition of the numbers shown. In one episode, the teacher is focused on the problem: 'What number is added to 3 to make 7?' She shows the class three open fingers on her hand as she asks this, pointing to the 3 on the circle rim, and then shows seven fingers as she indicates the need to make 7 , pointing to the 7 in the centre. Some children are seen counting out seven on their fingers. When no correct answers are forthcoming from the class, the following exchange takes place:

Teacher: $\quad$ Make 7 with your fingers. [Shows seven fingers on her hands and several learners seen showing seven fingers] Now hide three fingers. [Teacher closes three fingers on her hand and asks class to do the same] Which number can we add with this 3 to make 7 ? [Teacher's hand shows four fingers remaining open] Now we made 7 and hide 3 , and what is left? The number that when we will add with 3 , we will get 7. [Teacher goes over and helps a child to close the same three fingers and asks her to count what is left]

Learner: 4. [Accepting 4, the teacher then counts out three toothpicks at the front as 1, 2, 3, then another 4 toothpicks as 1, 2, 3, 4. She then counts them altogether from 1-7]

Prior to and following this episode, we see instances of some learners able to give correct answers. However, we also see several learners who appear unaware of how many fingers to open, and what to do once they have one of the given numbers showing. Here, a stated problem that is given in terms of missing addends comes to be 'funnelled' into a subtraction problem through a transformation step and associated explanation, and then verified by adding the two numbers as an addition problem. The teacher appears aware of the equivalence between missing addend problems and subtraction, but this equivalence is not established for learners; rather, the equivalence is simply assumed, and subsequently verified empirically. Thus, a problem stated in terms of missing addends is worked out in terms of subtraction-based transformation activity, and checked through addition. Essentially, the sum below is presented as the stated problem to be solved (though not in this form):

$$
3+\square=7
$$

whilst the transformation activity instead involves solving the following subtraction problem:

$$
7-3=\square
$$

It could be argued that in transformation terms nothing has gone wrong here, but given that we see several learners filling gaps in the follow-up missing addend individual activity, with 11 at the centre, simply by adding the two numbers seen, there is evidence that neither the missing addend nor the subtraction routine have actually taken 'hold' for broad swathes of the class. Instead, the predominant interpretation of the problem involves 'adding' the two numbers that can be seen, a 'putting together' of the visual instruction to add with the numbers seen. Here, we see transformation activity which, whilst connected in mathematically coherent terms to the input object, does not work to establish this connection. The switch to subtraction is followed as an embodied imitation in the teacher's presence, but no explanation for establishing this switch as valid is provided; at the same time, no interim representational supports or associated explanations that link more directly to the stated problem are enacted. Essentially, referring back to the literature, whilst the disciplinary explanation is coherent, an instructional explanation is lacking.

\section{Episode 3}

In this episode, drawn from Davis (2010), a Grade 10 teacher is working on integer addition sums, such as: $-7+5$. Davis describes the teacher's instructions to the class as follows (p. 384):

Teacher: $\quad$ So if the signs are the same ... what do you do? ... You take the common sign ... and then ... you add. ... If the signs are not the same ... what do you do? You subtract.

Learners: [Chorus] Subtract.

Teacher: But first you take the sign of the what? The sign ... of the bigger number. You look at the bigger number between the two ... and then you take the sign ... of the bigger number.

Learners: [Chorus] Yes.

Teacher: This should always be the case.

As was the case in Episode 1, an ordered set of instructions is relayed to the class - 'first you take ... and then you take ...'. Some conditions for the application of transformation sequences are established at the outset: essentially ways to distinguish the input representation in order to recognise which transformation must be selected. Davis (2010), discussing this episode in terms of operations (addition) and objects (integers), notes that:

The regulative criteria required by the procedure indicate that the teacher and learners do not operate directly on the mathematical objects and relations being indexed (integer sums). They operate, instead on more familiar and intuitive objects and relations ('whole number' sums). (p. 385)

In terms of our analytical concepts, $-7+5$ is the input representation that is transformed through a series of TA steps that provide an algorithm for solving the problem. In the interim stages, following the instructions would produce these representations: 
Teacher: Calculate the answer to the sum [SP]: $-7+5=[\mathrm{IR}]$

If the signs are not the same ... what do you do? You subtract. [TA step 1]

[Following this TA step would result in the following interim representation]: $7-5=2$

You look at the bigger number between the two ... and then you take the sign ... of the bigger number. [TA step 2]

[Following this TA step would result in the following final representation]: -2

In this episode, the backgrounding of the input representation is more significant than in the previous two episodes. Within the transformation sequence that is presented, negative numbers simply do not figure. Further, whilst the transformation sequence is driven by the need to solve the given problem, the algorithm presented enacts steps that produce an interim representation that is not equivalent to the input object $(-7+5)$, even though equivalence with the input representation returns at the final stage. Essentially, at the interim stage, transformation activity does not establish 'reason-able' connections between representations. As noted already, it could be argued that this is not problematic as a mnemonic device, given that correct answers across the range of input representations specified can be reliably produced. However, analyses of South African performance on TIMMS items (Howie, 2003) and our own data (Adler, 2011) point to multiple basic errors in the realm of both integer calculations and algebraic manipulations requiring knowledge of integer sum transformations. Further, Thompson et al. (1994) note that the orientation to 'answer-getting' tends to work most reliably only for the learners 'who understood the problem in the first place, and understood it in such a way that the proposed sequence of operations fits their conceptualisation of the problem' (p. 9).

In terms of our concepts, the input representation coheres with the stated problem, but the interim representation produced through the first transformation step does not retain equivalence with the input representation, even though this equivalence is recovered at the final stage.

\section{Episode 4}

Venkat and Mhlolo (2011) present an incident involving a Grade 11 teacher working on a data-handling problem based on the data table shown in Table 1, the input representation introduced in the lesson.

In the process of asking generally about ways in which data can be presented, a student mentions the notion of a 'tally table'. Venkat and Mhlolo note the subsequent return by the teacher, after several interim episodes focused on a range of other stated problems, to the notion of tallying. The teacher shifts attention from a focus on the meaning of the frequency values in Table 1 with the following question:

OK before we move on, somebody talked about tally OK. Does anyone know how to tally the number 8 ?... Do you know or you want to try?

Having asked the question, she then adds a further column to her frequency table and gives it the title 'tallies'. She then shows the class how to tally the number ' 8 ', this being the
TABLE 1: Data table.

\begin{tabular}{cc}
\hline No. of children in the family & Frequency \\
\hline 0 & 8 \\
1 & 14 \\
2 & 20 \\
3 & 17 \\
4 & 10 \\
5 & 11 \\
\hline
\end{tabular}

Source: Venkat, H., \& Mhlolo, M.K. (2011). Objects and operations in mathematics teaching - extending our understanding of breakdowns. In T. Mamiala, \& F. Kwayisi (Eds.), Proceedings of the 19th Annual Meeting of the Southern African Association for Research in Mathematics, Science and Technology Education (pp. 246-259). Mafikeng: SAARMSTE

first frequency value in her table. Then, pausing to ask the class if they have seen this (pointing to her tally) before, she explains further and demonstrates:

OK so it's one, it's two, it's three, it's four and what happens to number five. [Indicates the diagonal line]. And then it's one, it's two and it's three. OK. Your tally and your frequencies must be of the same number.

In this episode we note that whilst transformation activity (producing the tally) does connect to the stated problem, the stated problem does not cohere with the frequency table as the input representation presented. Thus, whilst equivalence is maintained, and indeed emphasised, in the teacher's discourse between the input representation's frequency values and the representation that is produced (the tallies), this equivalence in the absence of coherence between the stated problem and the input representation tends to nullify the equivalence that is produced.

Table 2 summarises the application of the analytical concepts applied to the four episodes.

\section{Discussion}

Looking across Table 2, we can see that in relation to the analytical framing of transformation activity developed, differences in the detail of access to the representations and transformations involved emerge. Significantly, some episodes reflect more serious disruption of mathematical coherence and connection than others. We contend that having the discursive resources to see and talk about these differences is enabling for our work in teacher education.

In Episode 1, all the criteria are answerable in the affirmative, suggesting that basic connections and coherence are in place. Nash's practices, we believe, mirror the kind of teaching referred to in our earlier discussion of the literature. The fact that Nash is viewed as successful, with learners under him viewed as performing successfully, backs up our claim of basic connections and coherence. Thus, whilst concepts in the literature may well describe Nash's practice as 'procedural', the connections and coherence we have identified appear to open up access to appropriate transformation activity.

In Episode 2, the key issue is that a missing addend problem is assumed to be solvable through manipulating the sum into subtraction format. Of course, this is mathematically correct, but the transformation from missing addend form to 
TABLE 2: Applying the analytical concepts.

\begin{tabular}{|c|c|c|c|c|}
\hline Concepts and framing questions & Episode 1 & Episode 2 & Episode 3 & Episode 4 \\
\hline Stated problem (SP) & $\begin{array}{l}\text { Producing graphs of linear } \\
\text { functions with equations in } \\
\text { non-standard form }\end{array}$ & Calculating missing addends & $\begin{array}{l}\text { Calculating integer sums involving } \\
\text { one positive and one negative } \\
\text { number }\end{array}$ & How to tally \\
\hline Transformation activity (TA) & $\begin{array}{l}\text { Put } x=0 \text { to get } y \text {-intercept; } y=0 \\
\text { to get } x \text {-intercept; plot points, } \\
\text { join them; you get the graph }\end{array}$ & Take 7 fingers, then hide 3 & $\begin{array}{l}\text { Drop the signs, take the smaller } \\
\text { from the larger and put the sign } \\
\text { of the larger against the answer }\end{array}$ & $\begin{array}{l}\text { Write one, one, one, one, then } \\
\text { a cross through; check that tally } \\
\text { and frequency show the same } \\
\text { number }\end{array}$ \\
\hline $\begin{array}{l}\text { Does IR cohere with the SP } \\
\text { by providing an appropriate } \\
\text { representation to transform? }\end{array}$ & $\begin{array}{l}\text { Yes } \\
3 x-2 y=6\end{array}$ & $\begin{array}{l}\text { Yes } \\
\text { Clock diagram allows link to } \\
\text { missing addend }\end{array}$ & $\begin{array}{l}\text { Yes } \\
-7+5\end{array}$ & $\begin{array}{l}\text { No } \\
\text { A frequency number, rather than } \\
\text { raw data, is given }\end{array}$ \\
\hline $\begin{array}{l}\text { Does TA produce representations } \\
\text { that connect with the IR at: } \\
\text { - interim stages } \\
\text { - final stage } \\
\text { in mathematical terms? }\end{array}$ & $\begin{array}{l}\text { Yes } \\
\text { Yes }\end{array}$ & $\begin{array}{l}\text { Yes } \\
\text { Yes }\end{array}$ & $\begin{array}{l}\text { No } \\
\text { Yes }\end{array}$ & $\begin{array}{l}\text { No } \\
\text { No }\end{array}$ \\
\hline $\begin{array}{l}\text { Does TA, with associated explanation } \\
\text { serve to establish connections } \\
\text { between its steps and the IR? }\end{array}$ & Yes & No & No & No \\
\hline
\end{tabular}

subtraction form needs to be established for learners through explanation and mediating representations that allow for the equivalence between the two forms to be appropriated. Instead, an assertion or an assumption of equivalence is presented, rather than an establishment of the equivalence. An outcome of the assumed equivalence appears, in this lesson, to be ongoing difficulties with task completion. There is a notable absence of the analogical representations that Rowland (2012) describes as important within instructional explanations: in this instance, representations that provide 'direct models' (Carpenter, Fennema, Franke, Levi \& Empson, 1999) of the stated missing addend problem. Thus, the MDI fails to provide representations that scaffold the connection between the missing addend situation and the abstract understanding of number relationships needed to 'jump' to subtraction as an appropriate transformation step to enact.

In Episode 3, the problem seen in Episode 2 is further compounded by the fact that the first transformation step indicated by the teacher's instructions produces an interim representation that does not connect with the input representation. Thus, the instruction that seeing one negative and one positive number means we have to subtract is arbitrary at this stage; it simply has to be remembered, and cannot be reasoned. Whilst at the final stage, equivalence with the input representation is resurrected, one needs to take on trust that this will happen through the interim working. As in Episode 2, the transformation activity does produce the correct answer with some efficiency. In this case though, connections between representations are broken at the interim stage.

In Episode 4, given that the stated problem is tallying, the presentation of a frequency table as the input representation is inappropriate as an object for the process of tallying to act on. Thus, whilst equivalence between the tally graphic produced for each frequency is maintained, connections that could serve to establish the purpose of tallying processes in mathematics are not simply made invisible, but actively disrupted.

\section{Conclusions}

Several comments emerge from our analysis. Firstly, we note at the most basic level that if the input representation does not cohere with the stated problem, this appears to negate the possibilities for answering the other analytical questions in the affirmative. At the intermediate level, we suggest that two criteria allow for further disaggregation:

- making transformation steps 'reason-able' by establishing connections between the representation and its transformation

- producing transformation sequences that connect across representations.

At the highest level, we have episodes that demonstrate coherence between the stated problem and the input representation, and connections between the representations produced through transformation activity where all three criteria are met.

Our sense is that the literature as it stands provides us with a discourse that can speak constructively to Nash's practice, but offers few insights into the kinds of limitations seen in our other episodes. The analytic concepts and questions that we have presented in this article were derived from analysing problematic episodes of the teaching of procedures involving the transformation of representations. Whilst the framework still needs further testing, our application of these concepts and criteria to further episodes from our project data sets suggests that they may provide some general principles for basic coherence and connection within mathematics teaching. We therefore offer the concepts and questions developed and deployed in this article as a starting point that has some generality, and illuminating potential for the many classrooms in which the transformation activity that fundamentally underlies mathematical activity still appears to be problematic.

\section{Acknowledgements}

This work is based upon the research of the Wits Mathematics Connect - Primary and Secondary Projects at the University 
of the Witwatersrand, supported, respectively by FirstRand Foundation, Anglo American, Rand Merchant Bank, the Department of Science and Technology, and the FirstRand Foundation (FRF) Mathematics Education Chairs Initiative of the FirstRand Foundation, the Department of Science and Technology (DST). Both are administered by the National Research Foundation (NRF). Any opinion, findings and conclusions or recommendations expressed in this material are those of the authors and do not necessarily reflect the views of the institutions named above.

\section{Competing interests}

We declare that we have no financial or personal relationship(s) which might have inappropriately influenced our writing of this article.

\section{Authors' contributions}

H.V. (University of the Witwatersrand) began the writing of the manuscript in terms of its framing, but all ongoing work has been shared by H.V. and J.A. (University of the Witwatersrand).

\section{References}

Adler, J. (2011). Research and Development Chairs: Annual report, 2010. Pretoria: National Research Foundation.

Adler, J. (2012). Knowledge resources in and for school mathematics teaching. In G Gueudet, B. Pepin, \& L. Trouche (Eds.), From text to 'lived' resources. Mathematic curriculum materials and teacher development (pp. 3-22). Dordrecht: Springer. http://dx.doi.org/2010.1007/978-94-007-1966-8_1

Adler, J., \& Pillay, V. (2007). An investigation into mathematics for teaching: Insights from a case. African Journal of Research in Mathematics, Science and Technology Education, 11(2), 87-108.

Artigue, M. (2011). Challenges in basic mathematics education. Paris: UNESCO. Available from http://unesdoc.unesco.org/images/0019/001917/191776e.pdf

Askew, M., Venkat, H., \& Mathews, C. (2012). Coherence and consistency in South African primary mathematics lessons. In T-Y. Tso (Ed.), Proceedings of the 36th Conference of the International Group for the Psychology of Mathematics Education, Vol. 2 (pp. 27-34). Taipei, Taiwan: PME.

Carpenter, T.P., Fennema, E., Franke, M.L., Levi, L., \& Empson, S.B. (1999). Children's mathematics: Cognitively guided instruction. Portsmouth, NH: Heinemann.

Davis, Z. (2010). Researching the constitution of mathematics in pedagogic contexts: From grounds to criteria to objects and operations. In V. Mudaly (Ed.), Proceeding of the 18th Annual Meeting of the Southern African Association for Research in Mathematics, Science and Technology Education, Vol. 1 (pp. 378-387). Durban: SAARMSTE. Available from http://www.sdu.uct.ac.za/usr/sdu/downloads/ conferences/saar_mste2010/longpapervol1.pdf

De Lima, R.N., \& Tall, D. (2008). Procedural embodiment and magic in linear equations. Educational Studies in Mathematics, 6(1), 3-18. http://dx.doi.org/10.1007/ s10649-007-9086-0
Department of Basic Education. (2011a). Report on the Annual National Assessments of 2011. Pretoria: DBE. Available from http://www.education. gov.za/LinkClick.asp $x$ ? fileticket=1U5igeVjiqg\%3d\&tabid=358\&mid $=1325$

Department of Basic Education. (2011b). Report on the National Senior Certificate examination results: 2010. Pretoria: DBE. Available from http://www.education. gov.za/LinkClick.aspx?fileticket=ah59UwgTOHk\%3d\&tabid=358\&mid=1325

Duval, R. (2006). A cognitive analysis of problems of comprehension in a learning of mathematics. Educational Studies in Mathematics, 61(1/2), 103-131. http:// dx.doi.org/10.1007/s10649-006-0400-z

Duval, R. (2008). Eight problems for a semiotic approach. In L. Radford, G. Schubring, \& F. Seeger (Eds.), Semiotics in mathematics education: Epistemology, history classroom and culture (pp. 39-61). Rotterdam: Sense Publishers.

Haapasalo, L. (2003). Linking procedural and conceptual mathematical knowledge in technology-based learning. In A. Rogerson (Ed.), Proceedings of the International Conference of the Mathematics Education into the 21st Century Project: The Decidable and the Undecidable in Mathematics Education (pp. 98-102). Brno, Czech Republic: Mathematics Education into the 21st Century Project. Available from http://math.unipa.it/ grim/21_project/21_brno03_Haapasalo.pdf

Haapasalo, L., \& Kadijevich, D. (2000). Two types of mathematical knowledge and their relation. Journal für Mathematik-Didaktik, 21(2), 139-157.

Hiebert, J., Gallimore, R., Garnier, H., Givvin, K.B., Hollingsworth, H., Jacobs, J., et al. (2003). Teaching mathematics in seven countries: Results from the TIMSS 1999 video study. Washington, DC: U.S. Department of Education, National Center for Education Statistics.

Howie, S.J. (2003). Language and other background factors affecting secondary pupils' performance in Mathematics in South Africa. African Journal of Research in Science, Mathematics and Technology Education, 7, 1-20.

Kieran, C. (2004). The core of algebra: Reflections on its main activities. In K. Stacey, H. Chick, \& M. Kendal (Eds.), The future of the teaching and learning of algebra. The 12th ICMI Study (pp. 21-34). Boston, MA: Kluwer Academic Publishers. http:// dx.doi.org/10.1007/1-4020-8131-6 2

Leinhardt, G. (2001). Instructional explanations: A commonplace for teaching and location for contrast. In V. Richardson (Ed.), Handbook for research on teaching (pp. 333-357). Washington, DC: American Educational Research Association.

Leinhardt, G., Zaslavsky, O., \& Stein, M.K. (1990). Functions, graphs and graphing Tasks, learning and teaching. Review of Educational Research, 60(1), 1-64.

Long, C. (2005). Maths concepts in teaching: Procedural and conceptual knowledge. Pythagoras, 62, 59-65. http://dx.doi.org/10.4102/pythagoras.v0i62.115

Mhlolo, M.K., Venkat, H., \& Schafer, M. (2012). The nature and quality of the mathematical connections teachers make. Pythagoras, 33(1), 1-9. http://dx.doi. org/10.4102/pythagoras.v33i1.22

Pillay, V. (2006). An investigation into mathematics for teaching: The kind of mathematical problem-solving teachers do as they go about their work. Unpublished master's thesis. University of the Witwatersrand, Johannesburg, South Africa. Available from http://wiredspace.wits.ac.za/handle/10539/2190

Rowland, T. (2012). Explaining explaining. In S. Nieuwoudt, D. Loubscher, \& H. Dreyer (Eds.), Proceedings of the 18th Annual National Congress of the Association for Mathematics Education of South Africa, Vol. 1 (pp. 54-66). Potchefstroom: AMESA. Available from http://www.amesa.org.za/AMESA2012/Volume1.pdf

Sekiguchi, Y. (2006). Coherence of mathematics lessons in Japanese eighth-grade classrooms. In J. Novotná, H. Moraová, M. Krátká, \& N.E. Stehlíková (Eds.) Proceedings of the 30th Conference of the International Group for the Psychology of Mathematics Education, Vol. 5 (pp. 81-88). Prague: PME.

Thompson, A.G., Philipp, R.A., Thompson, P.W., \& Boyd, B.A. (1994). Calculational and conceptual orientations in teaching mathematics. In D. Aichele, \& A. Coxford (Eds.), Professional development for teachers of mathematics (pp. 79-92). Reston VA: NCTM. Available from http://www.patthompson.net/PDFversions/1994Calc\& ConcOrientations.pdf

Venkat, H., \& Mhlolo, M.K. (2011). Objects and operations in mathematics teaching - extending our understanding of breakdowns. In T. Mamiala, \& F. Kwayisi (Eds.), Proceedings of the 19th Annual Meeting of the Southern African Association for Research in Mathematics, Science and Technology Education (pp. 246-259). Mafikeng: SAARMSTE. 